\title{
Selective Lymphocyte Activation and Inhibition of In Vitro Tumor Cell Growth by Novel Morphinans
}

\author{
${ }^{1,2}$ Ricardo Gomez-Flores, ${ }^{2}$ Kimberly R. Vietti, ${ }^{3}$ William J. Dunn III, ${ }^{1}$ Reyes Tamez-Guerra and ${ }^{2}$ Richard J. \\ Weber \\ ${ }^{1}$ Universidad Autónoma de Nuevo León, Facultad de Ciencias Biológicas, Departamento de Microbiología \\ e Inmunología, San Nicolás de los Garza, Nuevo León, México \\ ${ }^{2}$ Department of Biomedical and Therapeutic Sciences, University of Illinois College of Medicine, Peoria, \\ Illinois, USA \\ ${ }^{3}$ University of Illinois College of Pharmacy, Department of Medicinal Chemistry and Pharmacognosy, \\ Chicago, Illinois, USA
}

\begin{abstract}
Opioids can suppress immune functions and increase susceptibility to developing cancer and infectious diseases. Recently, novel opioid compounds have been synthesized that lack immunosuppressive effects. We evaluated the effects of morphinans with substituted pyrimidine (methyl, phenyl, hydroxy, and amino groups) and pyrazole groups on in vitro rat thymic lymphocyte and splenic macrophage functions, and tumor cell growth. We observed that morphinans with methyl, phenyl, hydroxy, amino, and pyrazole groups at concentrations from $10^{-10} \mathrm{M}$ to $10^{-5} \mathrm{M}$ plus Con $\mathrm{A}(2.5$ $\mu \mathrm{g} / \mathrm{ml}$ ) significantly $(\mathrm{P}<0.01$ ) induced 2- to 2.9-, 2.3- to 6.4-, 2.4- to 3.4-, 2.6- to 3.4-, and 2.6- to 3.2fold increases respectively in thymic lymphoproliferation compared with Con A alone; this effect was reversed by naloxone. Macrophage nitric oxide production was not altered by morphinans. In addition, we observed that all tested morphinans were associated with significant $(\mathrm{P}<0.01)$ in vitro tumor cell growth inhibition of J774A (18-41\%), L929 (12-36\%), L5178 (9-15\%) cell lines in a dose-dependent manner, at doses ranging from $10^{-11} \mathrm{M}$ to $10^{-5} \mathrm{M}$. Morphinans may be applied in clinical situations where immunosuppression is undesirable.
\end{abstract}

Key words: Rodent, spleen, thymus, monocytes/macrophages, $\mathrm{T}$ lymphocytes, nitric oxide, tumor immunity.

\section{INTRODUCTION}

Opioids represent a major source of relief for most chronic moderate to severe nonmalignant pain $^{[1]}$. However, they can alter immune function and consequently increase susceptibility to developing cancer and infectious diseases ${ }^{[2-4]}$. Novel opioid compounds have been synthesized that have analgesic capacity, but lack immunosuppressive effects or even potentiate immune function ${ }^{[5-9]}$. In this respect, Nowak et al. (1998) reported that the non-peptide agonist SNC80 did not alter NK cell, lymphocyte, and macrophage functions following intracerebroventricular administration $^{[7]}$. However, intravenous administration of SNC80 was associated with ex vivo immunopotentiation $^{[5]}$, and significant in vitro stimulation of lymphoproliferation (up to $174 \%$ stimulation) of resident and concanavalin A (Con A)treated lymphocytes ${ }^{[9]}$. Furthermore, SNC80 was recently shown to increase survival of tumor-bearing mice and significantly reduce tumor size ${ }^{[10]}$. In addition, novel non-peptide naltrindole and naltrexone derivatives, and the tetrahydroquinoline CGPM-9 have shown immunoenhancing properties in vitro ${ }^{[6,8,9]}$. Nonpeptide opioid agonists have proven to overcome major disadvantages of peptidic opioids (rapid degradation and their low potential to cross the blood brain barrier), because they are not only highly selective and potent, but are also proteolytically stable, thus increasing their clinical applications ${ }^{[11]}$. In addition, their usefulness as non-immunosuppressive analgesics has been demonstrated $^{[12]}$.

The $\mu$-opioid receptor selective morphinans, such as levallorphan, cyclorphan and butorphanol, are oxymorphon derivatives first introduced by Grewe in 1946. They are similar in structure to the morphine analogues, but lack the $\mathrm{E}$ ring found in the naturally occurring opioids, as well as the $6-\mathrm{OH}$ and the 7,8-double bond. Although $\mu$ agonists have been associated with immunosuppression $^{[3,13-16]}$ several novel non-peptide opioid compounds have shown immunopotentiating effects $^{[5,6,8,9-11]}$. 
The present study was undertaken to evaluate the structure and function relationship of the non-peptidic opioid morphinans on in vitro lymphocyte and macrophage functions, and in vitro tumor cell growth. We found that depending upon their structure, morphinans differentially enhanced lymphoproliferation without altering macrophage functions, and inhibited in vitro cell growth of various human and murine tumor cell lines.

\section{MATERIALS AND METHODS}

Reagents and culture media: Penicillin-streptomycin solution, L-glutamine, RPMI 1640, and AIM-V media were obtained from Life Technologies (Grand Island, NY). Morphinans with substituted pyrimidino (methyl, phenyl, hydroxy, and amino groups) and pyrazole groups (Fig. 1) were synthesized by William J. Dunn III from the Department of Medicinal Chemistry and Pharmacognosy, University of Illinois College of Pharmacy, Chicago, Illinois. Concanavalin A (Con A), LPS from Escherichia coli serotype 0128:B12, DMSO, HCl, MTT, red blood cell lysing buffer, sodium dodecyl sulfate (SDS), dimethylformamide (DMF), and fetal bovine serum were purchased from Sigma Chemical Co. (St. Louis, MO).

Animals: Fischer 344N male rats (200-220g) were purchased from Harlan Sprague-Dawley Inc. (Indianapolis, IN). They were housed in a pathogen free environment, 2-4 animals per cage, fed an ad libitum diet of laboratory food pellets and water and were maintained on a schedule of 12 hours light (0600-1800) and 12 hours dark (1800-0600) at $21 \pm 3{ }^{\circ} \mathrm{C}$. Animals were euthanized by asphyxiation in $100 \% \mathrm{CO}_{2}$ chamber.

Drug preparation: Morphinans were prepared and diluted to appropriate concentrations in AIM-V medium.

Cell preparation and culture: Single-cell suspensions were prepared by disrupting the spleen and thymus in RPMI 1640 medium. Cell suspensions were then washed three times in this medium, suspended and adjusted at appropriate densities with AIM-V medium, which has been observed to support cell culture ${ }^{[17]}$.

T cell proliferation assay: $\mathrm{T}$ cell proliferation was determined by $\left[{ }^{3} \mathrm{H}\right]$-thymidine uptake as previously reported $^{[18]}$. Immediately after rat death, single-cell thymus suspensions were prepared as described above, and adjusted to $1 \times 10^{7}$ cells $/ \mathrm{ml}$. Cell suspensions (100 $\mu \mathrm{l})$ were added to round-bottomed 96-well plates (Becton Dickinson) containing triplicate cultures (100 $\mu \mathrm{l}$ ) of AIM-V medium (unstimulated control) or the mitogen Con $A$ at submaximal and maximal concentrations of $0.625,1.25$, and $2.5 \mu \mathrm{g} / \mathrm{ml}$ in the presence or absence of morphinans. After incubation for $44 \mathrm{~h}$ at $37^{\circ} \mathrm{C}$ with $5 \% \mathrm{CO}_{2},\left[{ }^{3} \mathrm{H}\right]$-methylthymidine (6.7 Ci/mmol, ICN Pharmaceuticals Inc., Costa Mesa, CA) was added $(1 \mu \mathrm{Ci} / 10 \mu \mathrm{l} /$ well $)$, and cultures were incubated for additional $4 \mathrm{~h}$. Cell cultures were then harvested with a semiautomatic cell harvester (Tomtec, Orange, CT), and cell-incorporated radioactivity was determined by liquid scintillation spectrophotometry using a Microbeta Plus liquid scintillation counter (model 1450, Wallac Oy, Turku, Finland) with a counting efficiency for tritium of $35 \%$. Proliferative responses of thymic lymphocyte to maximal and submaximal concentrations of Con A were used for data analysis.

Nitrite determination: Accumulation of nitrite in the supernatants of macrophage cultures was used as an indicator of nitric oxide production by resident or activated cells ${ }^{[19]}$. One hundred-microliter splenic cell suspensions, prepared as explained above, were adjusted to $1 \times 10^{7}$ cells $/ \mathrm{ml}$ in AIM-V medium and plated in flat-bottomed 96-well plates (Becton Dickinson) for $2 \mathrm{~h}$ at $37^{\circ} \mathrm{C}$. Non-adherent cells were then removed, $50 \mu \mathrm{l}$ of red cell lysing buffer (Sigma) was added to all wells, followed by the addition of 50 $\mu \mathrm{l}$ of AIM-V medium. After removal of the supernatant, adherent cells (about $10 \%$ of the input cells) were then incubated overnight in AIM-V medium in the presence or absence of morphinans. After incubation, macrophage monolayers were washed once in this culture medium. The final cell monolayers consisted of $>95 \%$ macrophages as judged by Giemsa's stain procedures. Morphinan-treated and untreated splenic macrophages were incubated in the presence or absence of LPS $(25 \mathrm{ng} / \mathrm{ml})$ in triplicate, in a total volume of $200 \mu \mathrm{l}$ AIM-V medium per well for 3 days. After incubation, supernatants were obtained and nitrite levels were determined with the Griess reagent ${ }^{[20]}$, using $\mathrm{NaNO}_{2}$ as standard. Optical densities at $540 \mathrm{~nm}$ were then determined in a microplate reader (Molecular Devices Corporation, Palo Alto, CA).

Antitumor activity: The effects of morphinans on in vitro growth of human and murine tumor cell lines of different lineage were determined. Lymphoid (L5178Y-R, JURKAT and H-9), myeloid (U937 and J774A-1) and fibroblastoid (L929) tumor cells were adjusted to $5 \times 10^{4}$ cell $/ \mathrm{ml}$, and were incubated in the presence or absence of morphinans for $44 \mathrm{~h}$. At the end of the incubation period, MTT (final concentration of $0.5 \mathrm{mg} / \mathrm{ml}$ ) was added to all wells, and cell cultures were incubated for additional $4 \mathrm{~h}$. After this, $100 \mu \mathrm{l}$ of cell lysing buffer $(20 \%$ SDS in $50 \%$ each DMF and water) was added to all wells, and cultures were incubated for $16 \mathrm{~h}$. Optical densities were then read at $540 \mathrm{~nm}$, and percentages of cell growth inhibition induced by morphinans compared to untreated control were calculated. 
Statistical analysis: The results were expressed as mean \pm SEM of the response of 4 separate rat tissues to each treatment (morphinan concentrations vs Con A concentrations) with 3 replicate determinations per treatment or experimental group (morphinan, Con A, morphinan \pm Con A, and LPS) from a representative experiment. All experiments were repeated at least three times with similar results. Level of significance was assessed by Student's $t$ test and analysis of variance (ANOVA).

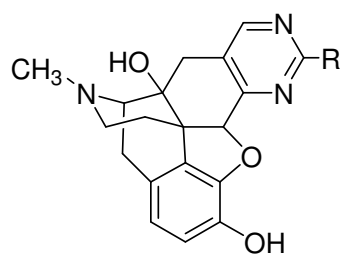

$$
\begin{aligned}
\text { 1a, } R=\mathrm{CH}_{3} \\
\text { 1b, } \mathrm{R}=\mathrm{C}_{6} \mathrm{H}_{5} \\
\text { 1c, } \mathrm{R}=\mathrm{OH} \\
\text { 1d, } \mathrm{R}=\mathrm{NH}_{2}
\end{aligned}
$$

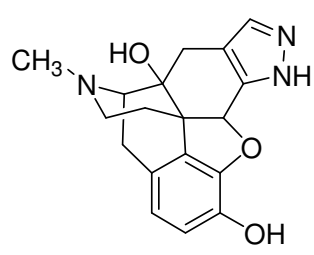

2.

Fig. 1: Morphinan structures.

\section{RESULTS}

Effect of morphinans on in vitro thymic lymphocyte proliferation: Morphinans with methyl, phenyl, hydroxy, amino, and pyrazole groups at concentrations from $10^{-10} \mathrm{M}$ to $10^{-5} \mathrm{M}$ plus Con A $(2.5 \mu \mathrm{g} / \mathrm{ml})$ significantly $(\mathbf{P}<0.01)$ induced 2 - to $2.9-$ - 2.3 - to $6.4-$, 2.4- to 3.4-, 2.6- to 3.4-, and 2.6- to 3.2-fold increases respectively in lymphoproliferation compared with Con A alone (Fig. 2). In addition, only the morphinan with the phenyl group (at $10^{-6} \mathrm{M}$ and $10^{-5} \mathrm{M}$ ) potentiated Con A-induced lymphocyte proliferation at Con A concentrations of 0.625 and $1.25 \mu \mathrm{g} / \mathrm{ml}$ (data not shown).

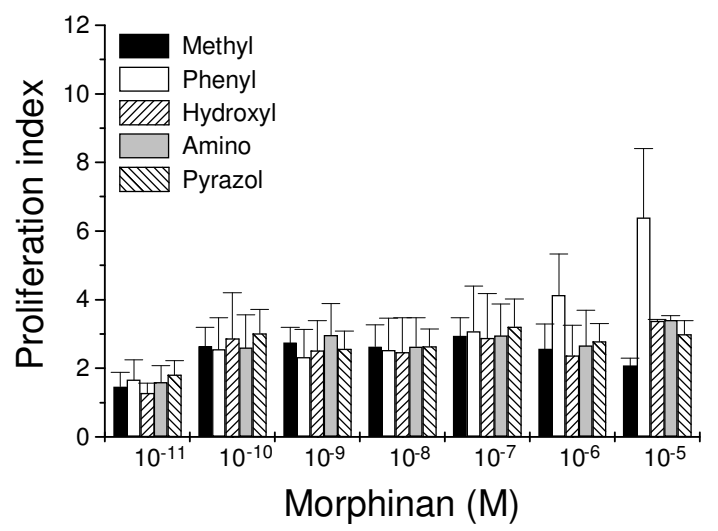

Fig. 2: Induction of lymphoproliferation by morphinans. Thymic cells were adjusted to $1 \times 10^{7}$ cells $/ \mathrm{ml}$, and incubated with Con A $(2.5 \mu \mathrm{g} / \mathrm{ml})$ in the presence or absence of morphinans for $48 \mathrm{~h}$ at $37^{\circ} \mathrm{C}$ in $5 \% \mathrm{CO}_{2} \cdot\left[^{3} \mathrm{H}\right]$ thymidine $(1 \mu \mathrm{Ci} /$ well $)$ was added $4 \mathrm{~h}$ prior to the end of the incubation period. Cell cultures were then harvested, and cell-incorporated radioactivity was determined as explained in the text. Proliferation index $=\mathrm{cpm}$ of morphinan plus Con A-treated cells/cpm of Con A-treated cells. Data represent mean \pm SEM of triplicates from a representative experiment. Thymic lymphocyte proliferation to Con A alone was $10440 \pm 1310 \mathrm{cpm}$.

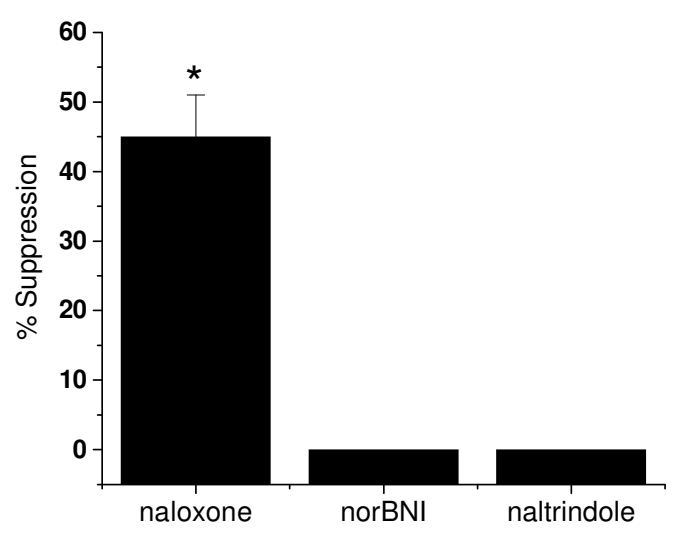

Fig. 3: Effect of opioid antagonists on morphinaninduced lymphoproliferation. Thymic cells were adjusted to $1 \times 10^{7} \mathrm{cells} / \mathrm{ml}$, and incubated with Con A $(2.5 \mu \mathrm{g} / \mathrm{ml})$ in the presence or absence of the morphinan with phenyl substituent at $10^{-5} \mathrm{M}$, with or without the antagonist at $10^{-5} \mathrm{M}$, for $48 \mathrm{~h}$ at $37^{\circ} \mathrm{C}$ in $5 \%$ $\mathrm{CO}_{2}$. $\left.{ }^{3} \mathrm{H}\right]$ thymidine $(1 \mu \mathrm{Ci} /$ well $)$ was added 4 $\mathrm{h}$ prior to the end of the incubation period. Cell cultures were then harvested, and cellincorporated radioactivity was determined as explained in the text. \% Suppression represents the reduction of morphinan plus Con Ainduced lymphoproliferation by the opioid antagonist. Data represent mean \pm SEM of triplicates from a representative experiment. $* \mathbf{P}<0.01$ compared with the effect of the morphinan alone. Thymic lymphocyte proliferation to Con A alone was $10440 \pm 1310$ cpm.

Morphinans alone did not stimulate lymphoproliferation. The effect of the most active of these compounds, the morphinan with a phenyl substituent, at $10^{-5} \mathrm{M}$ (a concentration that induced the highest lymphoproliferation stimulus (Fig. 2)) plus Con A $(2.5 \mu \mathrm{g} / \mathrm{ml})$, was antagonized by naloxone $(45 \%$ inhibition of morphinan plus Con A-induced lymphoproliferation at $10^{-5} \mathrm{M}$ ), but not by norbinaltorphimine and naltrindole (Fig. 3). 
Effect of morphinans on in vitro production of nitric oxide by splenic macrophages: Mophinans were observed not to alter nitric oxide production by splenic macrophages as shown in Fig. 4.

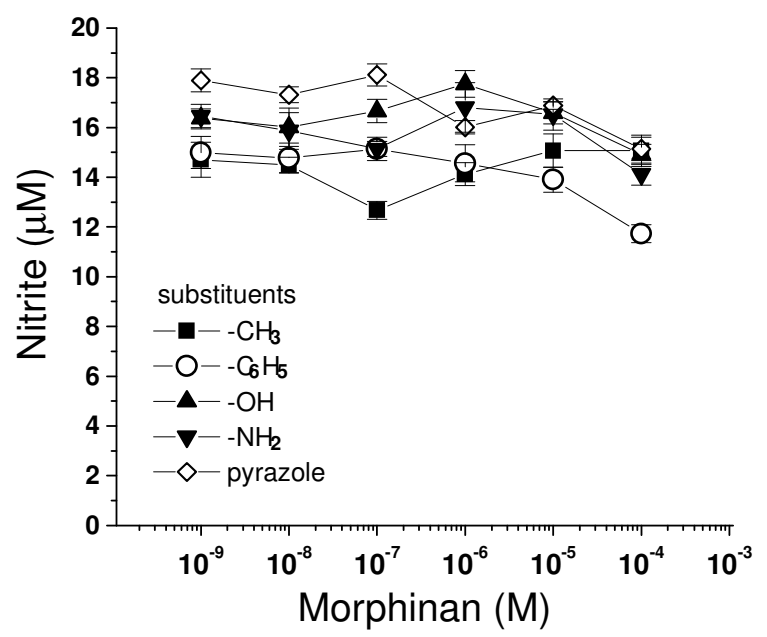

Fig. 4: Effect of morphinans on nitric oxide production by macrophages. One hundred-microliter splenic cell suspensions, were adjusted to $1 \times 10^{7}$ cells $/ \mathrm{ml}$ in AIM-V medium and incubated for $2 \mathrm{~h}$ at $37^{\circ} \mathrm{C}$. Non-adherent cells were then removed, and adherent cells (about $10 \%$ of the input cells or $1 \mathrm{x}$ $10^{6}$ cells $/ \mathrm{ml}$ ) were then incubated overnight in AIM-V medium in the presence or absence of morphinans. After incubation, macrophage monolayers were washed once in this culture medium. Morphinan-treated and untreated splenic macrophages were incubated in the presence or absence of LPS $(25 \mathrm{ng} / \mathrm{ml})$ in triplicate in AIM-V medium per well for 3 days. After incubation, supernatants were obtained and nitrite levels were determined with the Griess reagent. Optical densities at $540 \mathrm{~nm}$ were then determined in a microplate reader. Data represent mean \pm SEM of triplicates from a representative experiment. LPStreated cells alone released $15.25 \pm 1.8 \mu \mathrm{M}$ nitrite, whereas nitrite release by untreated macrophages was negligible.

Antitumor activity of morphinans: All tested morphinans (only data from phenyl-substituted morphinan is shown) were associated with significant $(\mathbf{P}<0.01)$ in vitro tumor cell growth inhibition of J774A (18-41\%), L929 (12-36\%), L5178 (9-15\%) cell lines in a dosedependent manner, at doses ranging from $10^{-11} \mathrm{M}$ to $10^{-5} \mathrm{M}$ (Fig. 5). However, morphinans marginally inhibited U937, H9 and Jurkat tumor cell growth (1\%-10\%).

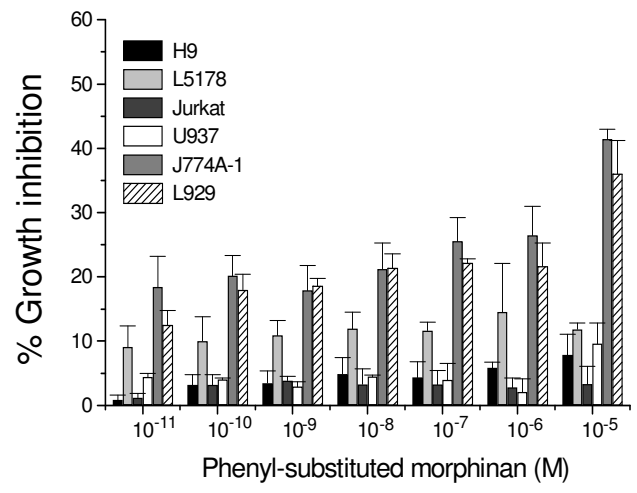

Fig. 5: Effect of morphinans on in vitro tumor cell growth. H9, L5178Y-R, JURKAT, U937, J774A-1, and L929 tumor cells were adjusted to $5 \times 10^{4} \mathrm{cell} / \mathrm{ml}$, and were incubated in the presence or absence of morphinan with phenyl substituent for $44 \mathrm{~h}$. At the end of the incubation period, MTT (final concentration of $0.5 \mathrm{mg} / \mathrm{ml}$ ) was added to all wells, and cell cultures were incubated for additional $4 \mathrm{~h}$. After this, $100 \mu \mathrm{l}$ of cell lysing buffer were added to all wells, and cultures were incubated for $16 \mathrm{~h}$. Optical densities were then read at $540 \mathrm{~nm}$, and percentages of cell growth inhibition induced by the morphinan compared to untreated control were calculated.

\section{DISCUSSION}

Non-peptidic opioids are highly selective, potent, and proteolytically stable ${ }^{[11]}$. Morphine represents the most widely utilized of these compounds and is clinically the opioid of choice for pain relief. However, morphine is also a well known immunosuppressive agent ${ }^{[13-16,21]}$. In previous reports, we showed that novel non-peptidic $\mu$ and $\delta$ opioids have the ability to potentiate immune function ${ }^{[5-8]}$. In the present study, we demonstrated that morphinans with substituted pyrimidine and pyrazole groups at concentrations of $10^{-}$ ${ }^{10}$ to $10^{-5} \mathrm{M}$ were associated with increased Con Astimulated rat thymic lymphocyte proliferation with the order of potency phenyl > pyrazole, hydroxy, amino > methyl > Con A alone (control). This effect was antagonized by naloxone, but not by norbinaltorphimine and naltrindole. These results indicate that the inclusion of a phenyl substituent at the 2' position of the pyrimidine group significantly potentiated Con A-induced thymic cell proliferation, and that this effect may be mediated through $\mu$-opioid receptor subtype. Opioids produce their effects in leukocytes via interactions with $\mu, \delta$, and $\kappa$ opioid receptors. These receptors exhibit different patterns of ligand selectivity, stereoselectivity, saturability and 
nanomolar affinity for opioids ${ }^{[22]}$. We observed that the effect of the most immunoactive of the morphinan compounds at $10^{-5} \mathrm{M}$ plus Con $\mathrm{A}(2.5 \mu \mathrm{g} / \mathrm{ml})$, was antagonized by naloxone ( $45 \%$ inhibition at equimolar concentration), but not by nor-binaltorphimine and naltrindole. This indicated that the morphinan action was mostly mediated through saturable $\mu$-opioid receptors, rather than kappa or delta opioid receptors, on the surface of the lymphocytes. Presence of opioid receptors on the surface of cells is not specific and absolute (lymphocytes do not possess $100 \%$ of opioid receptors of a particular type). In fact, they will have receptors for $\mu, \kappa$, and $\delta$ opioids at different densities. The use of antagonists at higher concentrations than the ligands (5 or 10 times the agonist concentration) has been tested in our laboratory with other opioids resulting in no further increase in the antagonist effect when utilized at equimolar concentration ${ }^{[6,8]}$. These facts may indirectly suggest that morphinans bind to selective, saturable, and high affinity membrane $\mu$ opioid receptors on leukocytes ${ }^{[22]}$. This is relevant for two major reasons: a) the $\mu$-receptor is considered to be the major site of analgesic action, since opioids used for pain management bind to this receptor with high affinity ${ }^{[23]}$, and b) current use of $\mu$ opioids such as morphine for pain control are also immunosuppressive $[13-16,21]$.

It was also demonstrated the morphinans did not alter macrophage functions, thus indicating a selective immunopotentiating effect on lymphocytes. Differential effects of opioids on leukocyte functions are commonly observed $^{[24,25]}$. In this regard, Kowalski et al. (1995) reported that enkephalins were associated with both suppressing and enhancing effects on splenic NK cell and macrophage functions related to the treatment period ${ }^{[24]}$. In addition, Pacifici et al. (1994) observed time-dependent biphasic effects of morphine, but not methadone, on immune parameters in vivo ${ }^{[25]}$. In yet other studies, dosedependent bimodal responses of lymphocytes and macrophages to opioids have been reported ${ }^{[26,27]}$, and Ryng et al. (1999) demonstrated that non-opioid substituted phenilamides of 5-amino-3-methylisooxazole4-carboxylic acid have differential effects on lymphocyte and macrophage functions ${ }^{[28]}$. In addition, Hicks et al. (2001) reported that the tetrahydroquinoline CGPM-9 enhanced rat thymic lymphoproliferation, but suppressed nitric oxide and tumor necrosis factor-alpha production by peritoneal macrophages ${ }^{[6]}$. In this latter study, the $\mu$ opioid receptor selective antagonist CTOP used at equimolar doses significantly suppressed the effect of CGPM-9 on lymphocyte and macrophage functions ${ }^{[6]}$. The mechanism (s) by which morphinans enhance lymphocyte proliferative response, but not alter macrophage functions, remains to be investigated. However, potentiating lymphocyte functions, while not affecting nitric oxide (NO) production by macrophages, may be advantageous for these opioid derivatives. NO is produced during inflammation and can be both beneficial and detrimental for the organism ${ }^{[29-32]}$. Although NO has been associated with antimicrobial and antitumor properties $^{[29,30]}$, it also induces immunosuppression by affecting lymphocyte and macrophage functions through direct action on these cells ${ }^{[31,32]}$, or indirectly via the central nervous system $(\mathrm{CNS})^{[33,34]}$. $\mathrm{NO}$ is also associated with CNS pathology ${ }^{[35]}$. In contrast, stimulation of lymphoproliferation by morphinan derivatives may be utilized in clinical situations where lymphocyte blood pool is significantly reduced, as in the cases of AIDS and aging $^{[36]}$. Therefore, morphinan derivatives may potentiate lymphoproliferative responses, without altering macrophage functions and thus avoiding potential pathological states.

Additionally, we showed that morphinans have the ability to significantly and selectively inhibit in vitro cell growth of murine tumor cells, and marginally inhibited growth of human tumor cells. However, the mechanism by which morphinans inhibit tumor cell growth remains to be elucidated. Selectivity of morphinans towards murine cells might be the result of some mechanism of drug resistance present in the human cell lines. Development of resistance to drugs may be related to failure of the cells to undergo apoptosis in response to the drug, failure of the drug to reach and/or affect its intracellular target, intracellular drug transport resistance mechanisms, subcellular redistribution, drug interaction, enhanced DNA repair, and failure to apoptose ${ }^{[37,38]}$.

Novel non-peptide opioids could potentially be utilized in many different clinical situations where immunosuppression is undesirable, as shown for $\mu$ selective ligands such as morphine ${ }^{[39]}$. Because of their effects on immune function, $\mu$-opioid agonists might not be optimal for management of moderate to severe pain following a variety of surgical procedures, cancer, and other related traumatisms. However opioid derivatives, such as morphinans, may have not only the potential to stimulate the immune system, but also have the capacity to inhibit tumor cell growth. Thus making these compounds potentially suitable in treating not only pain, but also enhancing the immune status of immunocompromised individuals against cancer and infectious diseases. Understanding structure and function relationship will allow the design, synthesis and use of non-immunosuppressive or immunostimulating opioids as adjuvants in the therapy of disease-associated pain in the individual with immune dysfunction.

\section{ACKNOWLEDEGEMENTS}

This study was supported by, respectively, CONACYT (National Council for Science and Technology of Mexico) and PAICYT (Science and Technology Research Supporting Program from Autonomous University of Nuevo Leon) grants I-32914-N and CN- 
285-00 to RGF, and by NIH Grants DA/AI0898, DA12095, DA08867, and F32-DA05865 to RJW.

\section{REFERENCES}

1. Passik, S.D. and H.J. Weinreb, 2000. Managing chronic nonmalignant pain: overcoming obstacles to the use of opioids. Adv. Ther., 17: 70-83.

2. Ishikawa, M.K., K. Tanno, A. Kamo, Y. Takayanagi and K. Sasaki, 1993. Enhancement of tumor growth by morphine and its possible mechanism in mice. Biol. Pharm. Bull., 16: 762766.

3. Gomez-Flores, R. and R.J. Weber, 1999. Opioids, opioid receptors, and the immune system. In: Cytokines-stress and immunity (eds N.P. Plotnikoff, R.E. Faith, A.J. Murgo and R.A. Good ) pp 281-314. Boca Raton, CRC Press.

4. Falek, A., R.M. Donahoe, J.J. Madden and D.A. Shafer, 1991. Opiates as immunosuppressive and genotoxic agents. Adv. Exp. Med. Biol., 288: 189201.

5. Gomez-Flores, R., K.C. Rice, X. Zhang and R.J. Weber, 2001. Increased tumor necrosis factor- $\alpha$ and nitric oxide production by rat macrophages following in vitro stimulation and intravenous administration of the non-peptidic $\tilde{\delta}$ opioid agonișt SNÇ80. Life Sci., 68: 2675-2684.

6. Hicks, M.E., R. Gomez-Flores, C. Wang, H.I. Mosberg and R.J. Weber, 2001. Differential effects of the novel non-peptidic opioid 4-tyrosylamido-6benzyl-1,2,3,4 tetrahydroquinoline (CGPM-9) on in vitro rat $\mathrm{T}$ lymphocyte and macrophage functions. Life Sci., 68: 2685-2694.

7. Nowak, J.E., R. Gomez-Flores, S.N. Calderon, K.C. Rice and R.J. Weber, 1998. Rat NK cell, T cell, and macrophage functions following intracerebroventricular injection of SNC 80. J. Pharmacol. Exp.Ther., 286: 931-937.

8. Riley, M.E., S. Ananthan and R.J. Weber, 1998. Novel non-peptidic opioid compounds with immunopotentiating effects. Adv. Exp. Med. Biol., 437: 183-187.

9. Caballero-Hernandez, D., R.J. Weber, M.E. Hicks, R. Tamez-Guerra, C. Rodriguez-Padilla, P. TamezGuerra, K.C. Rice, S. Ananthan, and R. GomezFlores, 2005. Potentiation of rat lymphocyte proliferation by novel non-peptidic synthetic opioids. Int. Immunopharmacol., 5: 1271-1278.

10. Gomez-Flores, R., D. Caballero-Hernandez, R. Tamez-Guerra, C. Rodriguez-Padilla, M.E. Hicks and R. Weber, 2005. Increased survival of tumorbearing mice by the delta opioid SNC80. Anticancer Res., 25: 3-7.

11. Hambrook, J.M., B.A. Morgan, M.J. Rance and C.F. Smith, 1976. Mode of deactivation of the enkephalins by rat and human plasma and rat brain homogenates. Nature, 262: 782-783.
12. Williams, W., K.C. Rice and R.J. Weber, 1991. Nonpeptide opioids: in vivo effects on the immune system. NIDA Res. Mon., 105: 404-407.

13. Gomez-Flores, R. and R.J. Weber, 1999. Inhibition of interleukin-2 production and downregulation of IL-2 and transferrin receptors on rat splenic lymphocytes following PAG morphine administration: a role in natural killer and $\mathrm{T}$ cell suppression. Cytokine Interferon Res., 19: 625630.

14. Rojavin, M., I. Szabo, J.L. Bussiere, T.J. Rogers, M.W. Adler and T.K. Eisenstein, 1993. Morphine treatment in vitro or in vivo decreases phagocytic functions of murine macrophages. Life Sci., 53: 997-1006.

15. Tubaro, E., U. Avico, C. Santiangeli, P. Zuccaro, G. Cavallo, R. Pacifici, C. Croce and G. Borelli, 1985. Morphine and methadone impact on human phagocytic physiology. Int. J. Immunopharmacol., 7: 865-874.

16. Gomez-Flores, R., J.L. Suo and R.J. Weber, 1999. Suppression of splenic macrophage functions following acute morphine action in the rat mesencephalon periaqueductal gray. Brain Behav. Immun., 13: 212-224.

17. Kaldjian, E.P., G.H. Chen and K.B. Cease, 1992. Enhancement of lymphocyte proliferation assays by use of serum-free medium. J. Immunol. Methods, 147: 189-195.

18. Gomez-Flores, R. and R.J. Weber, 2000. Differential effects of buprenorphine and morphine on immune and neuroendocrine functions following acute administration in the rat mesencephalon periaqueductal gray. Immunopharmacology, 48: 145-156.

19. Gomez-Flores, R., R. Tamez-Guerra, S.D. Tucker and R.T. Mehta, 1997. Bidirectional effects of IFNgamma on growth of Mycobacterium avium complex in murine peritoneal macrophages. J. Interferon Cytokine Res., 17: 331-336.

20. Green, L.C., D.A. Wagner, J. Glogowski, P.L. Skipper, J.S. Wishnok and S.R. Tannenbaum, 1982. Analysis of nitrate, nitrite, and $\left[{ }^{15} \mathrm{~N}\right]$ nitrate in biological fluids. Anal. Biochem., 126: 131-138.

21. Roy, S., R.A. Barke and H.H. Loh, 1998. MUopioid receptor-knockout mice: role of mu-opioid receptor in morphine mediated immune functions. Brain Res. Mol. Brain Res., 61: 190-194.

22. Lang, M.E., D. Jourd'Heuil, J.B. Meddings and M.G. Swain, 1995. Increased opioid binding to peripheral white blood cells in a rat model of acute cholestasis. Gastroenterology, 108: 1479-1486.

23. Raynor, K., H. Kong, A. Mestek, L.S. Bye, M. Tian, J. Liu, L. Yu and T. Reisine, 1995. Characterization of the cloned human mu opioid receptor. J. Pharmacol. Exp. Ther., 272: 423-428.

24. Kowalski, J., D. Belowski and J. Wielgus, 1995. Bidirectional modulation of mouse natural killer cell and macrophage cytotoxic activities by enkephalins. Polish J. Pharmacol., 47: 327-331. 
25. Pacifici, R., G. Patrini, I. Venier, D. Parolaro, P. Zuccaro and E. Gori, 1994. Effect of morphine and methadone acute treatment on immunological activity in mice: pharmacokinetic and pharmacodynamic correlates. J. Pharmacol. Exp. Ther., 269: 1112-1116.

26. Bessler, H., M.B. Sztein and S.A. Serrate, 1990. Beta-endorphin modulation of IL-1-induced IL-2 production. Immunopharmacology, 19: 5-14.

27. Van den, B.P., R. Dobber, S. Ramlal, J. Rozing and L. Nagelkerken, 1994. Role of opioid peptides in the regulation of cytokine production by murine CD4+ T cells. Cell. Immunol., 154: 109-122.

28. Ryng, S., M. Zimecki, Z. Sonnenberg and M.J. Mokrosz, 1999. Immunomodulating action and structure-activity relationships of substituted phenylamides of 5-amino-3-methylisoxazole-4carboxylic acid. Arch. Pharm. (Weinheim), 332: 158-162.

29. MacMicking, J., Q.W. Xie and C. Nathan, 1997. Nitric oxide and macrophage function. Annu. Rev. Immunol., 15: 323-350.

30. Cantuaria, G., A. Magalhaes, R. Angioli, L. Mendez, R. Mirhashemi, J. Wang, P. Wang, M. Penalver, H. Averette and P. Braunschweiger, 2000. Antitumor activity of a novel glyco-nitric oxide conjugate in ovarian carcinoma. Cancer, 88 : 381-388.

31. Albina, J.E., S. Cui, R.B. Mateo and J.S. Reichner, 1993. Nitric oxide-mediated apoptosis in murine peritoneal macrophages. J. Immunol., 150: 50805085 .
32. Stein, C.S. and G.H. Strejan, 1993. Rat splenocytes inhibit antigen-specific lymphocyte proliferation through a reactive nitrogen intermediate (RNI)dependent mechanism and exhibit increased RNI production in response to IFN-gamma. Cell. Immunol., 150: 281-297.

33. Wrona, D., 2006. Neural-immune interactions: An integrative view of the bidirectional relationship between the brain and immune systems. J. Neuroimmunol., 172: 38-58.

34. Raber, J. and F.E. Bloom, 1994. IL-2 induces vasopressin release from the hypothalamus and the amygdala: role of nitric oxide-mediated signaling. J. Neurosci., 14: 6187-6195.

35. Lewen, A., P. Matz, and P.H. Chan, 2000. Free radical pathways in CNS injury. J. Neurotrauma., 17: 871-890.

36. Haynes, B.F. and L.P. Hale, 1999. Thymic function, aging, and AIDS. Hosp. Pract., 34:59-60, 63-5, 69-70.

37. Ross, D.D., 2000. Novel mechanisms of drug resistance in leukemia. Leukemia, 14: 467-473.

38. Luqmani, Y.A., 2005. Mechanisms of drug resistance in cancer chemotherapy. Med. Princ. Pract., 14 (1): 35-48.

39. Lysle, D.T., M.E. Coussons, V.J. Watts, E.H. Bennett and L.A. Dykstra, 1993. Morphineinduced alterations of immune status: dose dependency, compartment specificity and antagonism by naltrexone. J. Pharmacol. Exp. Ther., 265: 1071-1078. 